\title{
Microscopic Description of Shape Transitions and Shape Coexistence in $\mathrm{Hg}$ Isotopes
}

\author{
V. Prassa ${ }^{1}$, K.E. Karakatsanis ${ }^{2,3}$ \\ ${ }^{1}$ Physics Department, University of Thessaly, Lamia GR-35100, Greece \\ ${ }^{2}$ Physics Department, Faculty of Science, University of Zagreb, \\ 10000 Zagreb, Croatia \\ ${ }^{3}$ Physics Department, Aristotle University of Thessaloniki, \\ Thessaloniki GR-54124, Greece
}

Received 14 October 2021

doi: https://doi.org/10.55318/bgjp.2021.48.5-6.495

\begin{abstract}
A microscopic analysis of characteristic signatures of nuclear ground-state shape transitions and coexisting nuclear shapes in neutron deficient even-even $\mathrm{Hg}$ isotopes is presented using a five dimensional collective Hamiltonian (5DCH) based on covariant densityfunctional theory. The triaxial deformation energy surfaces suggest coexisting configurations in ${ }^{190} \mathrm{Hg}, \gamma$-soft potential energy surfaces in ${ }^{192-198} \mathrm{Hg}$ and a more spherical structure in ${ }^{200} \mathrm{Hg}$. The corresponding $5 \mathrm{DCH}$ model calculations confirm the structural evolution in this region and suggest more increased collectivity than what can be deduced from the data.
\end{abstract}

KEY WORDS: covariant density functionals, collective states, shape transitions, $\mathrm{Hg}$ isotopes.

\section{Introduction}

Shape evolution in atomic nuclei is one of the most studied phenomena in low energy nuclear physics. When a major shell (single or doubly closed-shell nuclei) is completely filled the large energy gap to the next one stabilises a spherical configuration, whereas the residual interaction of the valence nucleons in openshell nuclei drive the nucleus toward deformed (quadrupole, octupole) equilibrium shapes. In a number of cases different shape configurations can coexist.

Extensive theoretical and experimental efforts were performed investigating the phenomena of phase transitions and phase coexistence in even-even nuclei near shell closures (see Refs. [1-6] for reviews). In the region of $Z=82$ near the neutron midshell $N=104$ the phenomena of phase coexistence [6] and phase transitions [7] were first observed in studies of hyperfine structure [8]. Later 
spectroscopic studies [9-18] revealed that the structure of those isotopes was defined by intruder prolate deformed configurations coexisting with less deformed oblate ground states. The low-lying excited states of the intruder band exhibit a parabola shape as a function of neutron number, starting from ${ }^{188} \mathrm{Hg}$ down to the midshell $N=104$, with a minimum observed at ${ }^{182} \mathrm{Hg}$ and going up to ${ }^{180} \mathrm{Hg}$ and ${ }^{178} \mathrm{Hg}[19,20]$. On the other hand, in the heavier transitional isotopes with $190<A<200$, the observed energy levels of the yrast band remain almost constant. Although, the isotopes between the stable ${ }^{200} \mathrm{Hg}$ and the beginning of the midshell in ${ }^{190} \mathrm{Hg}$ have been investigated by different experiments [21-32], there are still crucial observables that remain to be measured.

Theoretical studies based on the Gogny [33-35], the relativistic mean field (RMF) interactions [36,37], and the Nilsson-Strutinsky method [38] have generally confirmed these experimental findings. A systematic study of the low-lying states in the lead region has been performed within the number and angularmomentum projected generator coordinate method with axial symmetry, employing the Skyrme energy density functional (EDF) [39]. Excitation energies, electromagnetic transition rates, deformation properties, and ground-state properties relevant to the shape coexistence in $\mathrm{Hg}$ isotopes, have been investigated using the interacting boson model (IBM) [40-42]. A recent study within the Elliott and the proxy-SU(3) models [43] suggests that the evolution of shape coexistence in the neutron deficient $\mathrm{Hg}$ isotopes is accompanied by a merging of the spin-orbit (SO) - like shell with the open harmonic oscillator (HO) shell [43].

In this contribution we present contrained SCMF calculations for even-even ${ }^{190-}{ }^{200} \mathrm{Hg}$ isotopes within the relativistic Hartree-Bogoliubov [44] method with the density-dependent point-coupling (DD-PC1) [45] energy density functional in the particle-hole channel and a separable pairing force [46] in the particleparticle channel. The DD-PC1 density functional has been successfully applied to various studies of nuclear structure phenomena related to quantum phase transitions [47-50], shape coexistence [51] and the effect of collective correlations on the ground state and fission properties of superheavy nuclei [52,53].

A five-dimensional collective Hamiltonian $(5 \mathrm{DCH})$ with quadrupole deformations as dynamical collective coordinates $[54,55]$ is used to calculate the lowenergy excitation spectrum and the $\mathrm{B}(\mathrm{E} 2)$ transitions rates of ${ }^{190-200} \mathrm{Hg}$ isotopes. The microscopic self-consistent solutions of deformation-constrained triaxial relativistic Hartree-Bogolyubov (RHB) calculations, the single-particle wave functions, occupation probabilities, and quasiparticle energies, are used to calculate the Hamiltonian parameters. The moments of inertia are calculated with the Inglis-Belyaev formula [56,57] and the mass parameters with the cranking approximation [58]. The collective potential is obtained by subtracting the zero-point energy corrections [58] from the total energy that corresponds to the solution of constrained triaxial SCMF calculations. The resulting collective potential and inertia parameters as functions of the collective coordinates determine the dynamics of the 5DCH. Calculations shown here have been partially presented in $[59,60]$. 
To illustrate the rapid change of equilibrium shapes in Figure 1 we present the potential energy surfaces of even-even ${ }^{190-200} \mathrm{Hg}$ within the SCMF framework

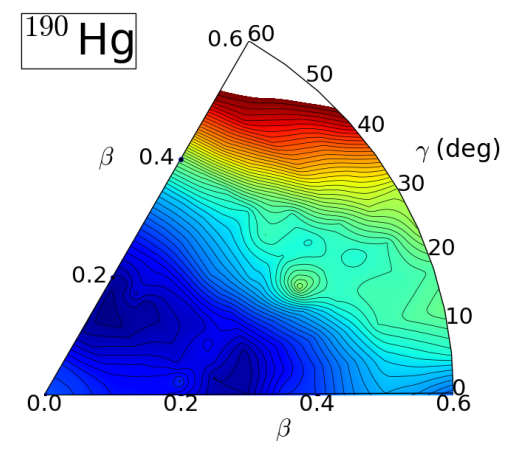

(a)

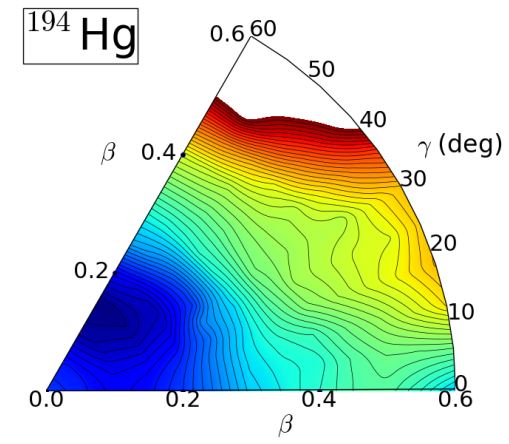

(c)

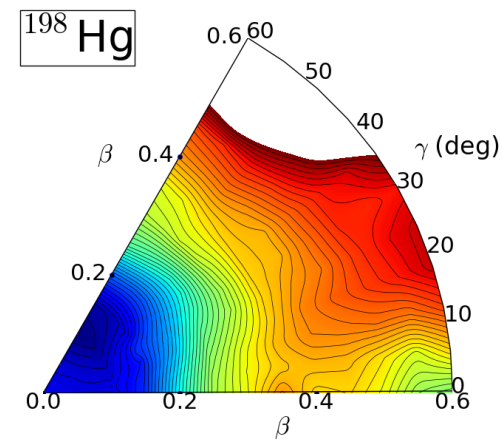

(e)

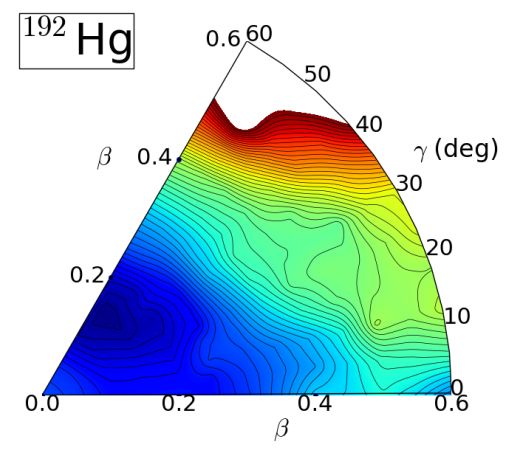

(b)

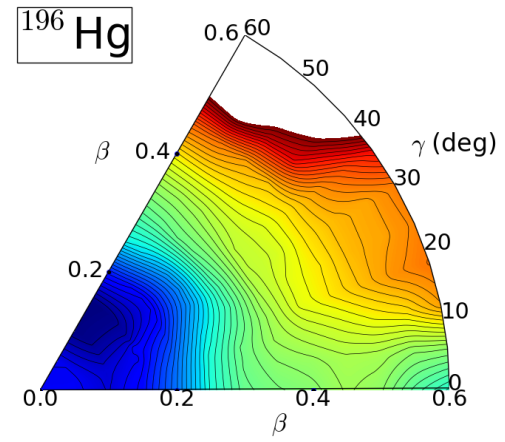

(d)

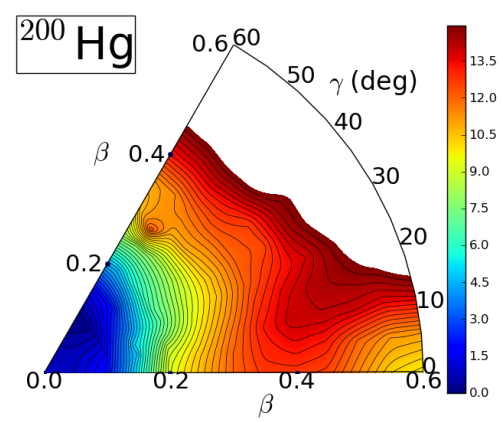

(f)

Figure 1. (Color online) Self-consistent RHB triaxial quadrupole energy surfaces of eveneven ${ }^{190-200} \mathrm{Hg}$ isotopes in the $\beta-\gamma$ plane $\left(0^{\circ}<\gamma<60^{\circ}\right)$. All energies are normalized with respect to the binding energy of the corresponding ground state. 
with the DD-PC1 functional and a separable pairing force. Starting with the lighter isotope ${ }^{190} \mathrm{Hg}$ the energy surface is $\gamma$-soft with two minima within an energy difference of $500 \mathrm{keV}$, which indicates a case of shape coexistence of the two different configurations. The more pronounced minimum is oblate deformed at $\beta \approx 0.15$ and the second one is prolate at $\beta \approx 0.25$. In ${ }^{192} \mathrm{Hg}$ the energy surface is still rather flat in the $\gamma$-direction with the equilibrium configuration on the oblate side at $0.1<\beta<0.2$. The prolate minimum diminishes and only the oblate one is seen in ${ }^{194-198} \mathrm{Hg}$. The single oblate minimum becomes less deformed and approaches $\beta=0$ for ${ }^{200} \mathrm{Hg}$, which implies a structural change from weakly oblate deformed to nearly spherical states.

The present calculations, based on the relativistic DD-PC1 functional, are consistent with other theoretical efforts in this region (using the interacting boson model based on the Gogny-D1M EDF [40], the D1 [33] and D1S [34,61] parametrizations of the Gogny-EDF, the Skyrme-SLy4 EDF [35],other Skyrme [39], the relativistic NL3 parametrization [36], and the relativistic PC-PK1 functional [62]).

\section{Spectroscopic Properties}

The constrained self-consistent solutions of the relativistic Hartree-Bogoliubov (RHB) equations at each point on the energy surface determine the mass parameters the three moments of inertia and the zero-point energy corrections as functions of the deformation parameters $\beta$ and $\gamma$. The diagonalization of the Hamiltonian yields the excitation spectra and collective wave functions that are used in the calculation of various observables, e.g., electromagnetic transition probabilities $\mathrm{B}(\mathrm{E} 2)$ and electric monopole transition strengths $\rho(E 0)$. Physical observables are calculated in the full configuration space and there are no effective charges in the model.

As an example in Figure 2 we display the low-lying collective spectrum of ${ }^{196} \mathrm{Hg}$, in comparison to available data for the excitation energies and reduced electric quadrupole transition probabilities B(E2) in Weisskopf units (W.u.) taken from Refs. [21]. In addition to the yrast ground-state band, in deformed and transitional nuclei excited states are also assigned to (quasi-) $\beta$ and $\gamma$-bands.

The comparison with the few existing experimental data shows a rather reasonable agreement of the excitation energy levels in the yrast band for $J^{\pi}<6^{+}$. The theoretical reduced electric-quadrupole transition probabilities $\mathrm{BE}(2)$ (in W.u.) are generally larger than the data. Although a reasonable agreement within the experimental errors is observed for the first excited state $2_{1}^{+}$, the calculated value for the $B\left(E 2 ; 4_{1}^{+} \longrightarrow 2_{1}^{+}\right)$overestimates the experimental value considerably. This indicates that there is probably more mixing between the theoretical states than what can be inferred from the data.

In addition we plot the probability distributions for the three band heads in the 


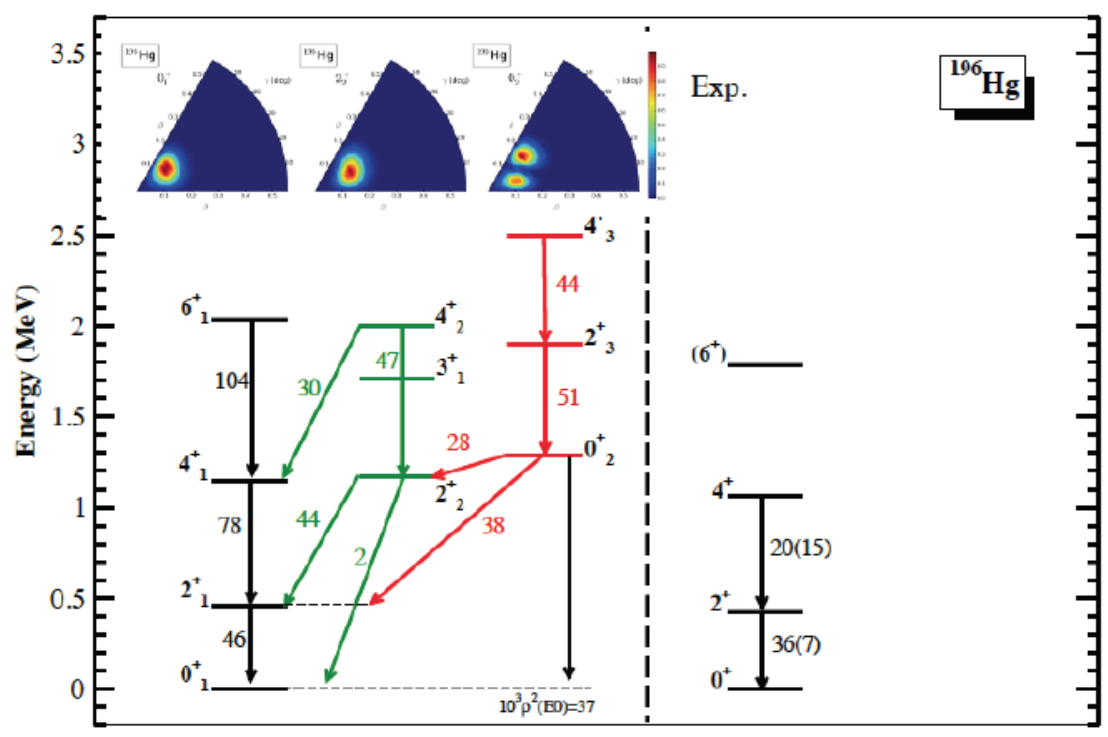

Figure 2. (Color online) Low-lying level scheme of the even-even ${ }^{196} \mathrm{Hg}$ nucleus. The excitation energies, the $\mathrm{B}(\mathrm{E} 2)$ values (in Weisskopf units) and the $\rho^{2}\left(E 0 ; 0_{2}^{+} \longrightarrow 0_{1}^{+}\right)$ obtained with the 5DCH based on the DD-PC1 functional are shown. Contour Plots: Probability density distributions in the $(\beta, \gamma)$ plane for the three band heads in the yrast band (left), the yrare band (middle), and the excited band built on the state $0_{2}^{+}$(right) in the even-even ${ }^{196} \mathrm{Hg}$ nucleus. The experimental data are taken from Ref. [21].

yrast band (left), the yrare band (middle), and the excited band built on the state $0_{2}^{+}$(right). The yrast state $0_{1}^{+}$in ${ }^{196} \mathrm{Hg}$ displays an oblate configuration with deformation around $0.1<\beta<0.2$ that extends in the $\gamma$-direction due to the $\gamma$ softness of the mean-field potential (cf. Figure 1). The $0_{2}^{+}$state has two peaks, a dominant one on the oblate side around $\beta \approx 0.2$ and a second one at smaller $\beta$ and $\gamma$ deformation of the same amplitude indicating that the $0_{2}^{+}$ state is presumably a $\beta$-vibrational state. The overlap between the $0_{1}^{+}$and $0_{2}^{+}$ states leads to level repulsion which probably causes the high excitation energy of the theoretical state $0_{2}^{+}$. The density distribution of the $2_{2}^{+}$state points to $\gamma$-vibrations in a triaxially deformed potential with a peak around $\gamma \approx 30^{\circ}$. Additionally, the close-lying doublet $3_{1}^{+}$and the $4_{2}^{+}$in the level scheme of ${ }^{196} \mathrm{Hg}$ (cf. Figure 2) is characteristic for a $\gamma$-band in a $\gamma$-soft potential. Both indicating that the $2_{2}^{+}$state is probably the band head of a (quasi) $\gamma$-band.

In Figure 3 we plot the theoretical values of $R_{4 / 2}$ as function of the neutron number of even-even ${ }^{190-200} \mathrm{Hg}$ isotopes in comparison to data taken from Ref. [21]. The calculated $R_{4 / 2}$ ratio starts at 2.32 in ${ }^{190} \mathrm{Hg}$ increases rapidly to 2.64 in ${ }^{192} \mathrm{Hg}$ and then decreases gradually to $2.45 \mathrm{in}{ }^{200} \mathrm{Hg}$. The experimental values in this region vary slighty around 2.5 as the neutron number increases. The crossing 


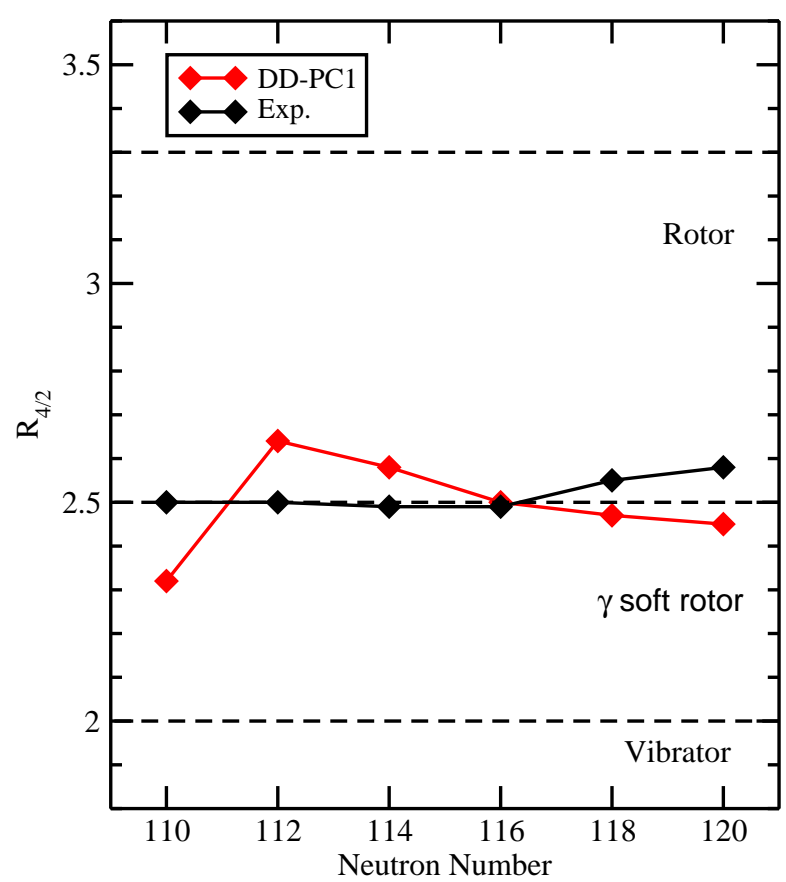

Figure 3. (Color online) The ratio $R_{4 / 2}$ of excitation energies of the yrast states $4_{1}^{+}$and $2_{1}^{+}$as a function of the neutron number in even-even ${ }^{190-200} \mathrm{Hg}$ isotopes calculated using the 5DCH model based on the DD-PC1 functional. The experimental data are taken from Ref. [21]

between the $2_{1}^{+}$and $4_{1}^{+}$normal and intruder states at $N=110 \mathrm{in} \mathrm{Hg}$ is probably the reason for the drop of the ratio $R_{4 / 2}$ in ${ }^{190} \mathrm{Hg}$, as reported in Ref. [42]. The results disclose the $\gamma$-softness of the potential energy surfaces in ${ }^{190-200} \mathrm{Hg}$. The strong configuration mixing predicted by the collective Hamiltonian model is probably the cause of the theoretical deviations from data.

In Figure 4 the calculated $B\left(E 2 ; 2_{1}^{+} \longrightarrow 0_{1}^{+}\right)$values (in Weisskopf units) within the 5DCH framework based on the DD-PC1 functional are shown. Theoretical results with the interacting boson model, IBM-2 [40] and the interacting boson model with configuration mixing (CM), IBM-CM [41] are presented for comparison. Our calculations reproduce the general decreasing trend with neutron number, however for all isotopes ${ }^{190-198} \mathrm{Hg}$ an increased collectivity in the $2_{1}^{+}$ yrast states is observed compared to data. For the isotopes ${ }^{192-200} \mathrm{Hg}$ the accuracy of the calculations compared to the experimental values is of the same quality as the one reached with the IBM-2 model in Ref. [40].

The most noticeable discrepancies between the theoretical calculations and the measured values are consistently in the lighter isotope ${ }^{190} \mathrm{Hg}$. The potential 


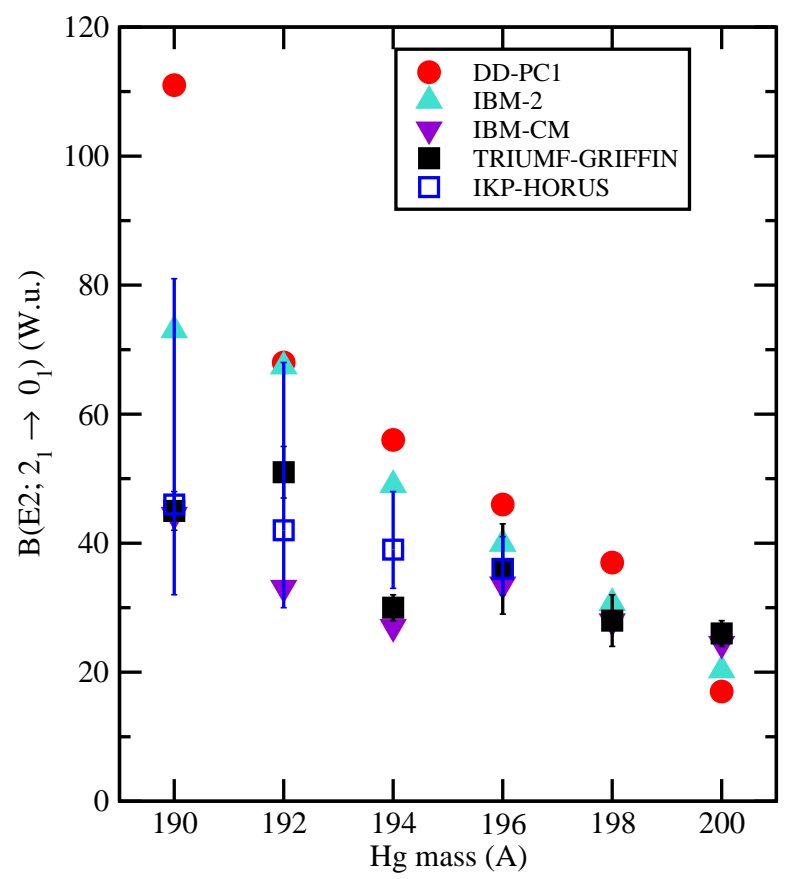

Figure 4. (Color online) $B\left(E 2 ; 2_{1}^{+} \longrightarrow 0_{1}^{+}\right)$values (in Weisskopf units) calculated using the $5 \mathrm{DCH}$ model with the DD-PC1 functional. Theoretical calculations with the IBM-2 [40], and IBM-CM [41] are given for comparison. Experimental values marked as TRIUMF-GRIFFIN are from Ref. [21] and those marked as IKP-HORUS are from Ref. [22].

energy surface of ${ }^{190} \mathrm{Hg}$ exhibits two minima, a dominant oblate configuration and a prolate one at larger defomation that are degenarate in energy with a rather flat path connecting them going through the triaxial region. The inclusion of dynamical correlations yields an oblate deformed but $\gamma$-soft $0_{1}^{+}$state and a $0_{2}^{+}$ state, within an energy gap of $400 \mathrm{keV}$, that is predominantly on the prolate side but with oblate admixtures. The relatively large overlap between the $0_{1}^{+}$and $0_{2}^{+}$wavefunctions, the large electric monopole transition strength $\rho(E 0)$ from the $0_{2}^{+}$to the $0_{1}^{+}$state $\left(\rho^{2}\left(E 0 ; 0_{2}^{+} \longrightarrow 0_{1}^{+}\right) \times 10^{3}=150\right)$ and the large $\mathrm{B}(\mathrm{E} 2)$ values of the interband transitions, suggest a strong mixing between the two configurations and support the hypothesis of shape-coexistence at $N=110$ in Hg.

\section{Overview and Conclusions}

Deformation constrained SCMF calculations have been performed with the relativistic Hartree-Bogoliubov method based on the universal energy density func- 
tional DD-PC1 and a separable pairing interaction. The triaxial $(\beta-\gamma)$ deformation energy surfaces obtained from the SCMF calculations for the even-even ${ }^{190-200} \mathrm{Hg}$ isotopes predict a very interesting nuclear structure evolution: coexistence of prolate and oblate deformed minima in ${ }^{190} \mathrm{Hg}, \gamma$-soft oblate deformed potential in ${ }^{192-198} \mathrm{Hg}$, and an almost spherical deformation in ${ }^{200} \mathrm{Hg}$.

A quadrupole collective Hamiltonian, with parameters determined by selfconsistent constrained triaxial RHB calculations, has been used to calculate the low-energy spectra, B(E2) transitions rates and electric monopole transition strengths $\rho(E 0)$ of $\mathrm{Hg}$ isotopes at neutron number $N=110-120$. The calculated excitations energies of the low-lying yrast band in ${ }^{196} \mathrm{Hg}$ reproduce the experimental values, however the $\mathrm{B}(\mathrm{E} 2)$ reduced transition probabilities for the $2_{1}^{+}$and $4_{1}^{+}$yrast states overestimate the data. The two low-lying bands based on the lowest excited vibrational state that appear in the energy spectrum support the hypothesis of increased collectivity in the theoretical calculations than what can be deduced from the data. The evolution with neutron number of the ratio $R_{4 / 2}$ validate the above assumptions. In ${ }^{190} \mathrm{Hg}$, the triaxial SCMF calculations of the energy surface and the results of the quadrupole collective Hamiltonian model suggest shape-coexistence of a dominant oblate configuration and a more prolate deformed intruder state.

Theoretical and experimental efforts in the region of even-even neutron deficient ${ }^{190-200} \mathrm{Hg}$ isotopes predict shape transitions from nearly spherical configurations in ${ }^{200} \mathrm{Hg}$ to $\gamma$-softness in ${ }^{192-198} \mathrm{Hg}$ and shape coexistence in ${ }^{190} \mathrm{Hg}$. The results presented in this work verify these findings, demonstrating the potential of the semi-empirical relativistic EDFs including the explicit treatment of collective correlations using a microscopic collective Hamiltonian.

\section{Acknowledgments}

V.P.'s research was conducted within the "Call Fellowship for Postdoctoral research" implemented by the University of Thessaly and funded by the Stavros Niarchos Foundation. The work of K.E.K. was financed within the Tenure Track Pilot Programme of the Croatian Science Foundation and the École Polytechnique Fédérale de Lausanne, and the Project No. TTP-2018-07-3554 Exotic Nuclear Structure and Dynamics with funds of the Croatian-Swiss Research Programme. Some of the results presented in this work have been produced using the AUTH Compute Infrastructure and Resources.

\section{References}

[1] K. Heyde, P. Van Isacker, M. Waroquier, J.L. Wood, and R.A. Meyer (1983) Phys. Rep. 102291.

[2] J.L. Wood, K. Heyde, W. Nazarewicz, M. Huyse, and P. van Duppen (1992) Phys. Rep. 215101. 
[3] A.N. Andreyev, et al. (2000) Nature (London) 405430.

[4] R. Julin, K. Helariutta, and M. Muikku (2001) J. Phys. G 27 R109.

[5] T. Grahn, A. Dewald, O. Möller, et al. (2008) Nucl. Phys. A 80183.

[6] K. Heyde and J.L. Wood (2011) Rev. Mod. Phys. 831467.

[7] P. Cejnar, J. Jolie, and R.F. Casten (2010) Rev. Mod. Phys. 822155.

[8] J. Bonn, G. Huber, H.-J. Kluge, L. Kugler, and E.W. Otten (1972) Phys. Lett. B 38 308.

[9] N. Rud, D. Ward, H.R. Andrews, R.L. Graham, and J.S. Geiger (1973) Phys. Rev. Lett. 311421.

[10] D. Proetel, R.M. Diamond, and F.S. Stephens (1974) Phys. Lett. B 48102.

[11] J.H. Hamilton, et al. (1975) Phys. Rev. Lett. 35562.

[12] J.D. Cole, et al. (1976) Phys. Rev. Lett. 371185.

[13] G.D. Dracoulis (2000) Phys. Scr. 200054.

[14] N. Bree, et al. (2014) Phys. Rev. Lett. 112162701.

[15] L.P. Gaffney, et al. (2014) Phys. Rev. C 89024307.

[16] R. Julin, T. Grahn, J. Pakarinen, and P. Rahkila (2016) J. Phys. G: Nucl. Part. Phys. 43024004.

[17] B.A. Marsh, T. Day Goodacre, et al. (2018) Nat. Phys. 141163.

[18] S. Sels, T. Day Goodacre, B.A. Marsh, et al. (2019) Phys. Rev. C 99044306.

[19] C. Müller-Gatermann, et al. (2019) Phys. Rev. C 99054325.

[20] J. Elseviers, et al. (2011) Phys. Rev. C 84034307.

[21] B. Olaizola, et al. (2019) Phys. Rev. C 100024301.

[22] A. Esmaylzadeh, et al. (2018) Phys. Rev. C 98014313.

[23] National Nuclear Data Center (NNDC), Nuclear Levels and Gamma Search.

[24] M.O. Kortelahti, et al. (1991) Phys. Rev. C 43484.

[25] A. Bockisch, K. Bharuth-Ram, A.M. Kleinfeld, and K.P. Lieb (1979) Z. Phys. A 289231.

[26] Th.J. De Boer, E.W. Ten Napel, and J. Block (1963) Physica 291013.

[27] M. Guttormsen, et al. (1983) Nucl. Phys. A 398119.

[28] R. Kroth, et al. (1980) Phys. Lett. B 97197.

[29] V. Kölschbach, et al. (1985)Nucl. Phys. A 439189.

[30] S.A. Hjorth, et al. (1980) Phys. Rev. Lett. 45878.

[31] R.M. Lieder, et al. (1975) Nucl. Phys. A 248, 317.

[32] R. Kroth, et al. (1981) Phys. Lett. B 99209.

[33] M. Girod and P.G. Reinhard (1982) Phys. Lett. B 1171.

[34] J.P. Delaroche, et al. (1994) Phys. Rev. C 502332.

[35] O. Moreno, P. Sarriguren, R. Álvarez-Rodríguez, and E. Moya de Guerra (2006) Phys. Rev. C 73054302.

[36] T. Nikšić, D. Vretenar, P. Ring, and G.A. Lalazissis (2002) Phys. Rev. C 65054320.

[37] R. Fossion, D. Bonatsos, and G.A. Lalazissis (2006) Phys. Rev. C 73044310.

[38] R. Bengtsson and W. Nazarewicz (1989) Z. Phys. A 334269.

[39] J.M. Yao, M. Bender, and P.-H. Heenen (2013) Phys. Rev. C 87034322.

[40] K. Nomura, R. Rodríguez-Guzmán, and L.M. Robledo (2013) Phys. Rev. C 87 064313. 
[41] J.E. García-Ramos and K. Heyde (2014) Phys. Rev. C 89014306.

[42] J.E. García-Ramos and K. Heyde (2018) EPJ Web of Conferences 17805005.

[43] A. Martinou, D. Bonatsos, T.J. Mertzimekis, K. Karakatsanis, I.E. Assimakis, S.K. Peroulis, S. Sarantopoulou, and N. Minkov (2021) Eur. Phys. J. A 5784.

[44] D. Vretenar, A. Afanasjev, G. Lalazissis, and P. Ring (2005) Phys. Rep. 409101.

[45] T. Nikšić, D. Vretenar, and P. Ring (2008) Phys. Rev. C 78034318.

[46] Y. Tian, Z.Y. Ma, and P. Ring (2009) Phys. Lett. B 67644.

[47] K. Nomura, T. Nikšić, and D. Vretenar (2016) Phys. Rev. C 94064310.

[48] K. Nomura, T. Nikšić, and D. Vretenar (2017) Phys. Rev. C 96014304.

[49] K. Nomura, D. Vretenar, T. Nikšić, and B.-N. Lu (2014) Phys. Rev. C 89024312.

[50] V. Prassa, T. Nikšić, and D. Vretenar (2013) Phys. Rev. C 88044324.

[51] Z.P. Li, T. Nikšić, and D. Vretenar (2016) J. Phys. G: Nucl. Part. Phys. 43024005.

[52] V. Prassa, T. Nikšić, G.A. Lalazissis, and D. Vretenar (2012) Phys. Rev. C 86 024317.

[53] Z. Shi, A.V. Afanasjev, Z.P. Li, and J. Meng (2019) Phys. Rev. C 99064316.

[54] Z.P. Li, T. Nikšić, D. Vretenar, P. Ring, and J. Meng (2010) Phys. Rev. C 81064321.

[55] T. Nikšić, D. Vretenar, and P. Ring (2011) Prog. Part. Nucl. Phys. 66519.

[56] D. Inglis (1956) Phys. Rev. C 1031786.

[57] S. Beliaev (1961) Nucl. Phys. A 24322.

[58] M. Girod and B. Grammaticos (1979) Nucl. Phys. A 33040.

[59] V. Prassa and K.E. Karakatsanis (2021) Int. J. Mod. Phys. E 30(7) 2150054. DOI: https://doi.org/10.1142/S0218301321500543.

[60] V. Prassa, K.E. Karakatsanis and G.A. Lalazissis (2021) EPJ Web of Conferences 252 02007. DOI: https://doi.org/10.1051/epjconf/202125202007.

[61] http://www-phynu.cea.fr/science_en_ligne/carte_potentiels_microscopiques/carte_ potentiel_nucleaire_eng.htm.

[62] X. Q. Yang, L.J. Wang, J. Xiang, X.Y. Wu, and Z.P. Li (2021) Phys. Rev. C 103 054321. 